\title{
G Gastos de salud según el tipo de seguro y la edad previa a la implementación de la cobertura universal de salud en Bolivia
}

\author{
Juan Pablo Jaldin ${ }^{1}$, Rodrigo K. Arce Cardozo ${ }^{2}$, Maria Kezia Lourdes Pormento
}

\section{Sra. Editora:}

A nosotros nos gustaría presenter un breve informe de estudio de gastos en salud segun el tipo de seguro y la edad, previa la implementación de la cobertura del seguro universal de salud en Bolivia. Este estudio nos ayudará a entender mejor sobre los gastos en salud de la población boliviana para continuar las investigaciones, y tomar acciones para mejorar el actual Sistema de salud.

\section{Introducción}

En Febrero de 2019, el gobierno Boliviano aprovó la cobertura universas de salud con la meta de proveer cobertura a la población desasegurada $(62,1 \%)$. A pesar de que hay literatura disponible, la cual evalua y describe el sistema de salud, no hay información en la distribución demografica sobre la mejora del servicio de cuidado de la salud ${ }^{1}$.

La evaluación sobre el monto de pago directo o la relación que tiene con el nivel de atención teniendo en cuenta las políticas de salud recientemente aprobadas, como el programa "familiar, comunitario e intercultural" (SAFCI), la expansión de beneficios y la creación de seguros de ancianos basados en el gobierno, y la ley 405 de la salud como un derecho humano $^{2}$. Las organizaciones internacionales han tratado de evaluar las diferencias en otros países de la región ${ }^{3,4}$ pero esta investigación se presenta a través de casos y no evalúa los sistemas de salud en su conjunto 5 . Es por esta razón que los investigadores evaluarán la distribución del gasto en salud contra el seguro de salud en Bolivia y su relación con todos los asegurados y no asegurados.

\section{Material y Metodos}

El conjunto de datos analizados es otorgado por el Instituto Nacional de Estadística (INE) del Gobierno Plurinacional de Bolivia y la información pública incluye la información de 35,693 hogares. La moneda boliviana se convirtió a dólares americanos para los fines del estudio. La encuesta se realizó del 21 de noviembre al 13 de diciembre de 2013. La metodología

${ }^{1}$ Medico, Universidad de San Andres Facultad de medicina

${ }^{2}$ Medico, NYU Langone Health, NYU Facultad de medicina, Departmento de salud publica

${ }^{3}$ Medico, Ateneo Facultad de medicina y salud publica

${ }^{\star}$ Correspondencia a: Juan Pablo Jaldin

Correo electrónico: dr.jpjaldin@gmail.com

Recibido el 08 de septiembre de 2019. Aceptado el 28 de septiembre de 2019. de la encuesta fue la entrevista cara a cara.

Chi-cuadrado se utilizó para ver variaciones entre nuestros grupos de interés utilizando SPSS. Análisis multivariado que evalúa las asociaciones entre el tipo de seguro de las personas entrevistadas y el porcentaje de gastos autoinformado en relación con los ingresos proporcionados.

\section{Resultados}

El gasto en salud se define como el porcentaje del ingreso gastado como hogar. Los resultados muestran que la mayoría de los encuestados no tienen seguro de salud 59,2\% (Tabla 1), independientemente de la edad, seguido de Seguridad Social $22 \%$, Público $17,1 \%$, Privado $1,5 \%$ y Otros $0,2 \%$. La mayoría de los encuestados gastaron menos del $10 \%$ de sus ingresos en salud y provenían del grupo de edad de 5 a 18 años. Los que gastaron $>160 \%$ no tenían seguro (Tabla 2).

\section{Discusión}

Los datos muestran que el seguro público tiene cierto éxito en mitigar los gastos en personas menores de 5 años y mayores de 61 años. Esto podría deberse a enfermedades prevalentes según la edad en ambos grupos, lo que se puede demostrar en futuras investigaciones. En otros grupos, los gastos son altos incluso en los asegurados, especialmente en el seguro de seguridad social. Esta categoría ha demostrado tener la mitad de los gastos en comparación con el grupo no asegurado después de ajustar por edad.

El número de personas sin seguro sigue siendo

Table 1. Tabla descriptive para sexo, edad y tipo de seguro

\begin{tabular}{llll}
\hline & & Cantidad & Column N \% \\
\hline \multirow{2}{*}{ Sexo } & Hombre & 17474 & $49.0 \%$ \\
& Mujer & 18219 & $51.0 \%$ \\
& Total & 35693 & $100.0 \%$ \\
\hline \multirow{2}{*}{ Edad } & $<5$ & 4259 & $11.9 \%$ \\
& $5-18$ & 9430 & $26.4 \%$ \\
\hline \multirow{2}{*}{ Tipo de seguro } & $19-30$ & 7229 & $20.3 \%$ \\
& $31-60$ & 11440 & $32.1 \%$ \\
& $>61$ & 3335 & $9.3 \%$ \\
& Total & 35693 & $100.0 \%$ \\
& Privado & 6101 & $17.1 \%$ \\
& Seguridad Social & 7843 & $1.5 \%$ \\
& Otros & 66 & $22.0 \%$ \\
& Ninguno & 21130 & $5.2 \%$ \\
& Total & 35693 & $100.0 \%$ \\
\hline
\end{tabular}


considerablemente alto e incluso si el gasto en salud de los pagos de bolsillo autoinformados es pequeño, esto no tiene en cuenta la necesidad de servicios, la pregunta permanece abierta si los segmentos de menores ingresos de la población son no poder pagar los servicios que requieren o necesitan.

Es necesario seguir explorando los condicionamientos que dan forma a la salud para sacar conclusiones definitivas.

Palabras clave: gastos de salud de bolsillo, seguro de salud, gastos de salud

\section{Referencias bibliográficas}

1. Ledo C, Soria R. Sistema de Salud de Bolivia. (Spanish). Health Syst Boliv Engl. 2011;53:S109S119.

2. Alvarez FN, Leys M, Mérida HER, Guzmán GE. Primary health care research in Bolivia: systematic review and analysis. Health Policy Plan. May 2015:czv013. doi:10.1093/heapol/czv013.

3. Woodhouse C, Lopez Camelo J, Wehby GL. A Comparative Analysis of Prenatal Care and Fetal Growth in Eight South American Countries. PLoS ONE. 2014;9(3):e91292. doi:10.1371/journal. pone.0091292.

4. Ramírez Hita S. [Intercultural aspects of the health system reform in Bolivia]. Rev Peru Med Exp Salud Pública. 2014;31(4):762-768.

5. WHO Ranking of Health System Performance. Science. 2001;293(5535):1595-1596.
Table 2. Tabulación cruzada de, edad, tipo de seguro y gastos

\begin{tabular}{|c|c|c|c|c|c|c|c|c|c|}
\hline \multirow{2}{*}{\multicolumn{4}{|c|}{ Gastos }} & \multicolumn{5}{|c|}{ Tipo de seguro } & \multirow{2}{*}{ Total } \\
\hline & & & & Publico & \multirow{2}{*}{$\begin{array}{c}\text { Privado } \\
62\end{array}$} & \multirow{2}{*}{$\begin{array}{c}\text { S, social } \\
768\end{array}$} & Otros & Ninguno & \\
\hline$<10 \%$ & Edad & $<5$ & Cantidad & 1814 & & & 6 & 1609 & 4259 \\
\hline & & & & $42,6 \%$ & $1,5 \%$ & $18,0 \%$ & $0,1 \%$ & $37,8 \%$ & $100,0 \%$ \\
\hline & & $5-18$ & Cantidad & 1057 & 124 & 1727 & 34 & 6384 & 9326 \\
\hline & & & $\begin{array}{l}\text { \% dentro de } \\
\text { la Edad }\end{array}$ & $11,3 \%$ & $1,3 \%$ & $18,5 \%$ & $0,4 \%$ & $68,5 \%$ & $100,0 \%$ \\
\hline & & $19-30$ & Cantidad & 838 & 122 & 1386 & 5 & 4610 & 6961 \\
\hline & & & $\begin{array}{l}\% \text { dentrode } \\
\text { la Edad }\end{array}$ & $12,0 \%$ & $1,8 \%$ & $19,9 \%$ & $0,1 \%$ & $66,2 \%$ & $100,0 \%$ \\
\hline & & $31-60$ & Cantidad & 1073 & 190 & 2733 & 11 & 6648 & 10655 \\
\hline & & & $\begin{array}{l}\text { \% dentro de } \\
\text { la Edad }\end{array}$ & $10,1 \%$ & $1,8 \%$ & $25,6 \%$ & $0,1 \%$ & $62,4 \%$ & $100,0 \%$ \\
\hline & & $>61$ & Cantidad & 951 & 24 & 799 & 8 & 791 & 2573 \\
\hline & & & $\begin{array}{l}\% \text { dentro de } \\
\text { la Edad }\end{array}$ & $37,0 \%$ & $0,9 \%$ & $31,1 \%$ & $0,3 \%$ & $30,7 \%$ & $100,0 \%$ \\
\hline & To & tal & Cantidad & 5733 & 522 & 7413 & 64 & 20042 & 33774 \\
\hline & & & $\begin{array}{l}\% \text { dentro de } \\
\text { la Edad }\end{array}$ & $17,0 \%$ & $1,5 \%$ & $21,9 \%$ & $0,2 \%$ & $59,3 \%$ & $100,0 \%$ \\
\hline $11-20 \%$ & Edad & $5-18$ & Cantidad & 2 & 1 & 1 & 0 & 19 & 23 \\
\hline & & & $\begin{array}{l}\text { \% dentro de } \\
\text { la Edad }\end{array}$ & $8,7 \%$ & $4,3 \%$ & $4,3 \%$ & $0,0 \%$ & $82,6 \%$ & $100,0 \%$ \\
\hline & & $19-30$ & Cantidad & 9 & 3 & 31 & 0 & 78 & 121 \\
\hline & & & $\begin{array}{c}\% \text { dentro de } \\
\text { la Edad }\end{array}$ & $7,4 \%$ & $2,5 \%$ & $25,6 \%$ & $0,0 \%$ & $64,5 \%$ & $100,0 \%$ \\
\hline & & $31-60$ & Cantidad & 27 & 7 & 70 & 0 & 212 & 316 \\
\hline & & & $\begin{array}{l}\text { \% dentro de } \\
\text { la Edad }\end{array}$ & $8,5 \%$ & $2,2 \%$ & $22,2 \%$ & $0,0 \%$ & $67,1 \%$ & $100,0 \%$ \\
\hline & & $>61$ & Cantidad & 96 & 2 & 76 & 1 & 88 & 263 \\
\hline & & & $\begin{array}{l}\% \text { dentro de } \\
\text { la Edad }\end{array}$ & $36,5 \%$ & $0,8 \%$ & $28,9 \%$ & $0,4 \%$ & $33,5 \%$ & $100,0 \%$ \\
\hline & To & tal & Cantidad & 134 & 13 & 178 & 1 & 397 & 723 \\
\hline & & & $\begin{array}{l}\% \text { dentro de } \\
\text { la Edad }\end{array}$ & $18,5 \%$ & $1,8 \%$ & $24,6 \%$ & $0,1 \%$ & $54,9 \%$ & $100,0 \%$ \\
\hline $21-40 \%$ & Edad & 5-18 & Cantidad & 2 & 0 & 5 & & 18 & 25 \\
\hline & & & & $8,0 \%$ & $0,0 \%$ & $20,0 \%$ & & $72,0 \%$ & $100,0 \%$ \\
\hline & & $19-30$ & Cantidad & 6 & 1 & 10 & & 44 & 61 \\
\hline & & & $\begin{array}{l}\% \text { dentro de } \\
\text { la Edad }\end{array}$ & $9,8 \%$ & $1,6 \%$ & $16,4 \%$ & & $72,1 \%$ & $100,0 \%$ \\
\hline & & $31-60$ & Cantidad & 9 & 1 & 35 & & 132 & 177 \\
\hline & & & $\begin{array}{l}\% \text { dentro de } \\
\text { la Edad }\end{array}$ & $5,1 \%$ & $0,6 \%$ & $19,8 \%$ & & $74,6 \%$ & $100,0 \%$ \\
\hline & & $>61$ & Cantidad & 67 & 2 & 53 & & 66 & 188 \\
\hline & & & $\begin{array}{l}\% \text { dentro de } \\
\text { la Edad }\end{array}$ & $35,6 \%$ & $1,1 \%$ & $28,2 \%$ & & $35,1 \%$ & $100,0 \%$ \\
\hline & To & tal & Cantidad & 84 & 4 & 103 & & 260 & 451 \\
\hline & & & $\begin{array}{l}\% \text { dentro de } \\
\text { la Edad }\end{array}$ & $18,6 \%$ & $0,9 \%$ & $22,8 \%$ & & $57,6 \%$ & $100,0 \%$ \\
\hline $41-80 \%$ & Edad & $5-18$ & Cantidad & 0 & 0 & 4 & 0 & 9 & 13 \\
\hline & & & $\begin{array}{l}\text { \% dentro de } \\
\text { la Edad }\end{array}$ & $0,0 \%$ & $0,0 \%$ & $30,8 \%$ & $0,0 \%$ & $69,2 \%$ & $100,0 \%$ \\
\hline & & $19-30$ & Cantidad & 2 & 4 & 9 & 0 & 26 & 41 \\
\hline & & & $\begin{array}{l}\% \text { dentro de } \\
\text { la Edad }\end{array}$ & $4,9 \%$ & $9,8 \%$ & $22,0 \%$ & $0,0 \%$ & $63,4 \%$ & $100,0 \%$ \\
\hline & & $31-60$ & $\begin{array}{l}\text { Cantidad } \\
\% \text { dentro de }\end{array}$ & 9 & 1 & 15 & 1 & 82 & 108 \\
\hline & & & la Edad & $8,3 \%$ & $0,9 \%$ & $13,9 \%$ & $0,9 \%$ & $75,9 \%$ & $100,0 \%$ \\
\hline & & $>61$ & Cantidad & 39 & 1 & 41 & 0 & 44 & 125 \\
\hline & & & $\begin{array}{l}\text { \% dentro de } \\
\text { la Edad }\end{array}$ & $31,2 \%$ & $0,8 \%$ & $32,8 \%$ & $0,0 \%$ & $35,2 \%$ & $100,0 \%$ \\
\hline & To & tal & Cantidad & 50 & 6 & 69 & 1 & 161 & 287 \\
\hline & & & $\begin{array}{l}\text { \% dentro de } \\
\text { la Edad }\end{array}$ & $17,4 \%$ & $2,1 \%$ & $24,0 \%$ & $0,3 \%$ & $56,1 \%$ & $100,0 \%$ \\
\hline $81-160 \%$ & Edad & $5-18$ & Cantidad & 1 & 0 & 4 & & 6 & 11 \\
\hline & & & $\begin{array}{l}\text { \% dentro de } \\
\text { la Edad }\end{array}$ & $9,1 \%$ & $0,0 \%$ & $36,4 \%$ & & $54,5 \%$ & $100,0 \%$ \\
\hline & & $19-30$ & Cantidad & 1 & 0 & 3 & & 10 & 14 \\
\hline & & & $\begin{array}{l}\text { \% dentro de } \\
\text { la Edad }\end{array}$ & $7,1 \%$ & $0,0 \%$ & $21,4 \%$ & & $71,4 \%$ & $100,0 \%$ \\
\hline & & $31-60$ & Cantidad & 6 & 1 & 8 & & 32 & 47 \\
\hline & & & $\begin{array}{l}\text { \% dentro de } \\
\text { la Edad }\end{array}$ & $12,8 \%$ & $2,1 \%$ & $17,0 \%$ & & $68,1 \%$ & $100,0 \%$ \\
\hline & & $>61$ & Cantidad & 24 & 4 & 13 & & 26 & 67 \\
\hline & & & $\begin{array}{l}\text { \% dentro de } \\
\text { la Edad }\end{array}$ & $35,8 \%$ & $6,0 \%$ & $19,4 \%$ & & $38,8 \%$ & $100,0 \%$ \\
\hline & Tot: & & Cantidad & 32 & 5 & 28 & & 74 & 139 \\
\hline & & & $\begin{array}{l}\% \text { dentro de } \\
\text { la Edad }\end{array}$ & $23,0 \%$ & $3,6 \%$ & $20,1 \%$ & & $53,2 \%$ & $100,0 \%$ \\
\hline$>160 \%$ & Edad & $5-18$ & Cantidad & 0 & & 6 & & 20 & 26 \\
\hline & & & $\begin{array}{l}\text { \% dentro de } \\
\text { la Edad }\end{array}$ & $0,0 \%$ & & $23,1 \%$ & & $76,9 \%$ & $100,0 \%$ \\
\hline & & $19-30$ & Cantidad & 2 & & 1 & & 5 & 8 \\
\hline & & & $\begin{array}{l}\% \text { dentro de } \\
\text { la Edad }\end{array}$ & $25,0 \%$ & & $12,5 \%$ & & $62,5 \%$ & $100,0 \%$ \\
\hline & & $31-60$ & Cantidad & 3 & & 5 & & 45 & 53 \\
\hline & & & $\begin{array}{l}\text { \% dentro de } \\
\text { la Edad }\end{array}$ & $5,7 \%$ & & $9,4 \%$ & & $84,9 \%$ & $100,0 \%$ \\
\hline & & $>61$ & Cantidad & 26 & & 16 & & 29 & 71 \\
\hline & & & $\begin{array}{l}\text { \% dentro de } \\
\text { la Edad }\end{array}$ & $36,6 \%$ & & $22,5 \%$ & & $40,8 \%$ & $100,0 \%$ \\
\hline & Tot: & & Cantidad & 31 & & 28 & & 99 & 158 \\
\hline & & & $\begin{array}{l}\% \text { dentro de } \\
\text { la Edad }\end{array}$ & $19,6 \%$ & & $17,7 \%$ & & $62,7 \%$ & $100,0 \%$ \\
\hline Total & Edad & $<5$ & Cantidad & 1814 & 62 & 768 & 6 & 1609 & 4259 \\
\hline & & & $\begin{array}{l}\% \text { dentro de } \\
\text { la Edad }\end{array}$ & $42,6 \%$ & $1,5 \%$ & $18,0 \%$ & $0,1 \%$ & $37,8 \%$ & $100,0 \%$ \\
\hline & & $5-18$ & Cantidad & 1062 & 125 & 1747 & 34 & 6456 & 9424 \\
\hline & & & $\begin{array}{l}\% \text { dentro de } \\
\text { la Edad }\end{array}$ & $11,3 \%$ & $1,3 \%$ & $18,5 \%$ & $0,4 \%$ & $68,5 \%$ & $100,0 \%$ \\
\hline & & $19-30$ & Cantidad & 858 & 130 & 1440 & 5 & 4773 & 7206 \\
\hline & & & 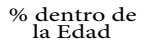 & $11,9 \%$ & $1,8 \%$ & $20,0 \%$ & $0,1 \%$ & $66,2 \%$ & $100,0 \%$ \\
\hline & & $31-60$ & Cantidad & 1127 & 200 & 2866 & 12 & 7151 & 11356 \\
\hline & & & $\begin{array}{l}\% \text { dentro de } \\
\text { la Edad }\end{array}$ & $9,9 \%$ & $1,8 \%$ & $25,2 \%$ & $0,1 \%$ & $63,0 \%$ & $100,0 \%$ \\
\hline & & $>61$ & Cantidad & 1203 & 33 & 998 & 9 & 1044 & 3287 \\
\hline & & & $\begin{array}{c}\% \text { dentro de } \\
\text { la Edad }\end{array}$ & $36,6 \%$ & $1,0 \%$ & $30,4 \%$ & $0,3 \%$ & $31,8 \%$ & $100,0 \%$ \\
\hline & Tot: & & Cantidad & 6064 & 550 & 7819 & 66 & 21033 & 35532 \\
\hline & & & $\begin{array}{l}\text { \% dentro de } \\
\text { la Edad }\end{array}$ & $17,1 \%$ & $1,5 \%$ & $22,0 \%$ & $0,2 \%$ & $59,2 \%$ & $100,0 \%$ \\
\hline
\end{tabular}

